\title{
Alloy-Electrode-Assisted High-Performance Enhancement-Type Neodymium-Doped Indium-Zinc-Oxide Thin-Film Transistors on Polyimide Flexible Substrate
}

\author{
Kuankuan Lu, Rihui Yao, Wei Xu, Honglong Ning $\mathbb{D}^{\mathrm{D}}$, Xu Zhang, Guanguang Zhang, Yilin Li, \\ Jinyao Zhong, Yuexin Yang, and Junbiao Peng
}

State Key Laboratory of Luminescent Materials and Devices, South China University of Technology, Guangzhou 510640, China

Correspondence should be addressed to Honglong Ning; ninghl@scut.edu.cn and Junbiao Peng; psjbpeng@scut.edu.cn

Received 2 November 2020; Accepted 1 March 2021; Published 22 March 2021

Copyright ( $) 2021$ Kuankuan Lu et al. Exclusive Licensee Science and Technology Review Publishing House. Distributed under a Creative Commons Attribution License (CC BY 4.0).

\begin{abstract}
Flexible thin-film transistors with high current-driven capability are of great significance for the next-generation new display technology. The effect of a Cu-Cr-Zr (CCZ) copper alloy source/drain (S/D) electrode on flexible amorphous neodymiumdoped indium-zinc-oxide thin-film transistors (NdIZO-TFTs) was investigated. Compared with pure copper (Cu) and aluminum (Al) S/D electrodes, the CCZ S/D electrode changes the TFT working mode from depletion mode to enhancement mode, which is ascribed to the alloy-assisted interface layer besides work function matching. X-ray photoelectron spectroscopy (XPS) depth profile analysis was conducted to examine the chemical states of the contact interface, and the result suggested that chromium (Cr) oxide and zirconium $(\mathrm{Zr})$ oxide aggregate at the interface between the S/D electrode and the active layer, acting as a potential barrier against residual free electron carriers. The optimal NdIZO-TFT exhibited a desired performance with a saturation mobility $\left(\mu_{\text {sat }}\right)$ of $40.3 \mathrm{~cm}^{2} \cdot \mathrm{V}^{-1} \cdot \mathrm{s}^{-1}$, an $I_{\text {on }} / I_{\text {off }}$ ratio of $1.24 \times 10^{8}$, a subthreshold swing (SS) value of $0.12 \mathrm{~V} \cdot \mathrm{dec}$ ade ${ }^{-1}$, and a threshold voltage $\left(V_{\text {th }}\right)$ of $0.83 \mathrm{~V}$. This work is anticipated to provide a novel approach to the realization of highperformance flexible NdIZO-TFTs working in enhancement mode.
\end{abstract}

\section{Introduction}

Today, $\mathrm{Cu}$ interconnection metallization, including bus lines and device electrodes, plays an important role in high refresh rate, ultra-high definition, and large size of flexible displays, owing to its low resistivity and good mechanical performance [1]. Although pure $\mathrm{Cu}$ has the advantages in terms of low resistivity $[2,3]$, it is difficult to be directly used as an electrode for a thin-film transistor (TFT) device due to its poor adhesion strength to a flexible substrate and copper atom diffusion issue. In view of this matter, copper alloying is a very promising copper metallization strategy without introducing a heterogeneous adhesion-barrier layer that leads to variety of etching problems in the subsequent photolithography process $[4,5]$. Conventional binary $\mathrm{Cu}$ alloys, such as $\mathrm{Cu}-\mathrm{Ca}[6]$, $\mathrm{Cu}-\mathrm{Mn}[7,8]$, and $\mathrm{Cu}-\mathrm{Ti}[9]$, have been verified to act as electrodes in TFTs preliminarily. However, the resistivity is uncompetitive due to the relatively high content of doped metals. In addition, polymer materials are diffusely used in optoelectronic technology, such as flexible substrate, conductor, insulator, and packaging materials [10-12]. Flexible substrates such as polyethylene naphthalate (PEN), polyethylene terephthalate (PET), polycarbonate (PC), and polyimide (PI) are widely tried to be used in flexible electronics due to their advantages of transparency, light weight, flexibility, and sturdiness $[13,14]$. Among them, PI is the most promising substrate for high-performance flexible display with $\mathrm{Cu}$ wiring because of its high thermal stability and good mechanical and chemical properties [15]. However, few studies specifically reported $\mathrm{Cu}$ alloy electrodes on PI substrates. As a result, low-resistivity $\mathrm{Cu}-\mathrm{Cr}-\mathrm{Zr}$ (CCZ) ternary alloy following the "microquantity and multielement" guideline was developed for a flexible PI substrate, which is proposed in our previous work [16].

Metal oxide materials have a wide range of electronical applications [17-20]. As an important component, amorphous metal oxide semiconductors (AOS) are developing rapidly in newly emerging application areas related to 


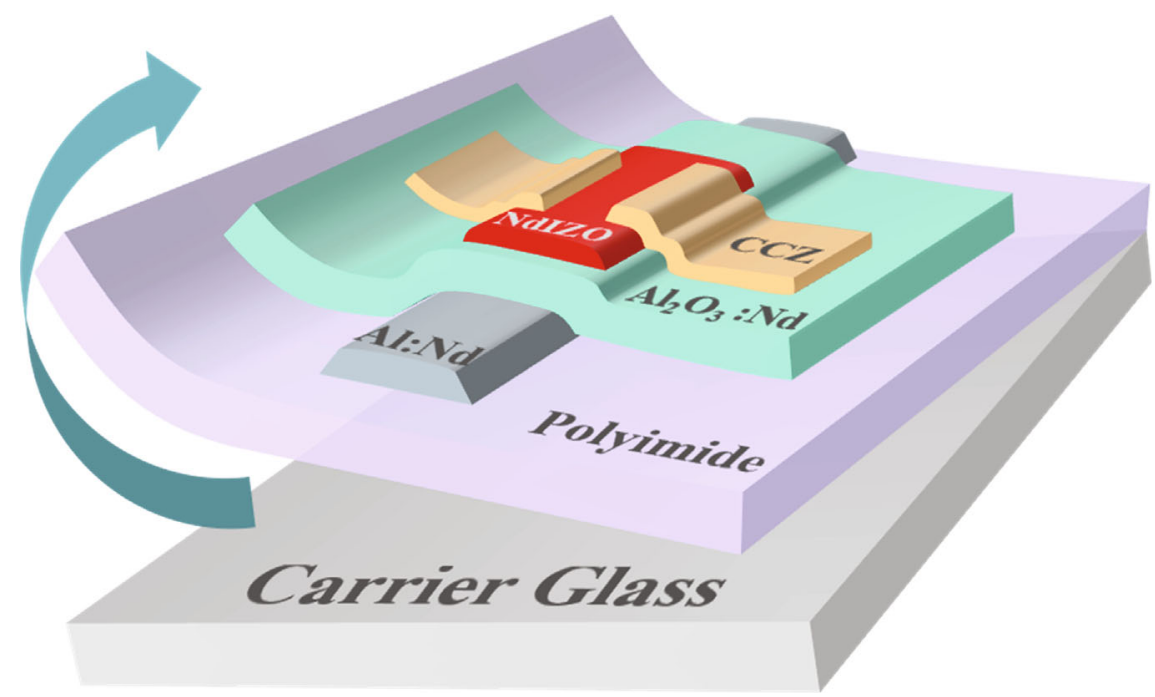

FIGURE 1: Structure diagram of a flexible thin-film transistor with CCZ S/D electrodes.

information display [21-24]. As a typical representative, indium- (In-) and zinc- ( $\mathrm{Zn-}$ ) based indium-gallium-zincoxide (IGZO) is a likely candidate to serve as the active layer of transistors in flexible display. Indeed, compared with conventional silicon- (Si-) based TFTs, AOSs exhibit attractive integrated electrical and optical properties such as good uniformity, high visible light transparency, high carrier mobility, low temperature preparation, and cost saving. The large spherical overlapping 5 s orbitals from In is of profound significance for the carrier migration mechanism, leading to the insensitivity of the carrier transport to the amorphous phase. Zn plays an important role in the enhancement of amorphization and modulates the shallow tail state [25]. Ga doped in IZO, though deteriorating the mobility performance, suppresses the generation of oxygen-deficient defects (i.e., oxygen vacancies $\left(V_{\mathrm{O}}\right)$ and metal interstitials), which improves the device stability [26]. As a matter of fact, aIGZO has been commercialized. Nevertheless, considering that the high-resolution backplane or high-framerate $3 \mathrm{D}$ display requires a mobility of $20-50 \mathrm{~cm}^{2} \cdot \mathrm{V}^{-1} \cdot \mathrm{s}^{-1}$, some issues of AOS such as low mobility (IGZO $\sim 10 \mathrm{~cm}^{2} \cdot \mathrm{V}^{-1} \cdot \mathrm{s}^{-1}$ ) and sensitivity to external environments are waiting to be resolved [27]. The insufficient mobility is due to the component of $\mathrm{Ga}$, which is so high that degrades the mobility dramatically $[28,29]$. In addition, $\mathrm{Ga}_{2} \mathrm{O}_{3}$ is acid-soluble and easy to be damaged in the wet etching process [30]. As a result, the carrier passivator, such as $\mathrm{Ta}, \mathrm{Zr}, \mathrm{Si}$, and Hf, was added into the matrix (IZO) to improve the stability performance [31-34]. It is of particular interest to note that a microquantity dopant can also make an enormous influence on the device stability without significant mobility deterioration, since the electrical behavior of the active layer is forcefully dependent on the carrier passivator, which is ascribed to the differences of the bonddissociation energy of the dopants [35]. Hence, the excessive carriers from $V_{\mathrm{O}}$ can be effectively alleviated by introducing more robust metal-oxygen bonds [36]. In comparison of all above carrier passivators, the rare earth element neodymium (Nd) is believed to be an excellent oxygen binder, which can suppress the generation of $V_{\mathrm{O}}$ and control the carrier concentration. In theory, lower work function metals such as $\mathrm{Al}, \mathrm{Ti}, \mathrm{Mo}$, or $\mathrm{Cu}$ are preferred as S/D electrodes to the formation of ohmic contact. Nevertheless, except for electrode work function, it has been confirmed that different types of electrodes lead to different contact resistances and interface electrical characteristics, which depend on the electronic structure and carrier density of the channel contact surface [37]. As a result, it is possible to modulate the carrier transport through chemical reactions with a specially designed alloy electrode.

In this paper, the contact characteristics of the interface between the CCZ S/D electrode and NdIZO active layer were investigated. The typical structure of the device is shown in Figure 1. Different electrodes, i.e., $\mathrm{CCZ}$, pure $\mathrm{Cu}$, and pure $\mathrm{Al}$, were utilized as the S/D electrode of the flexible NdIZOTFTs, and the element diffusion as well as the chemical states was studied by XPS analysis. The work function match between different materials was demonstrated by Ultraviolet Photoelectron Spectrometer (UPS)/Ultraviolet-visible Spectrophotometer (UV-Vis) tests, which makes it possible to depict the energy band diagram of the carrier transport at the interface between different S/D electrodes and the NdIZO active layer.

\section{Results and Discussion}

2.1. The Electrical Performance of TFTs with Different S/D Electrodes. Figure 2 shows the electrical characteristics of the NdIZO-TFTs with different S/D electrodes under optimal annealing temperature of $360^{\circ} \mathrm{C}$, that is, the value of $I_{\mathrm{D}}$ (drain current) as a function of $V_{\mathrm{G}}$ (gate voltage) or $V_{\mathrm{D}}$ (drain voltage). As shown in Figures 2(a)-2(c), devices possess reliable reproducibility. Particularly, the NdIZO-TFTs with the CCZ S/D electrode (TFT-CCZ) have the best reproducibility, which reveals better stability than pure metals such as $\mathrm{Cu}$ and 


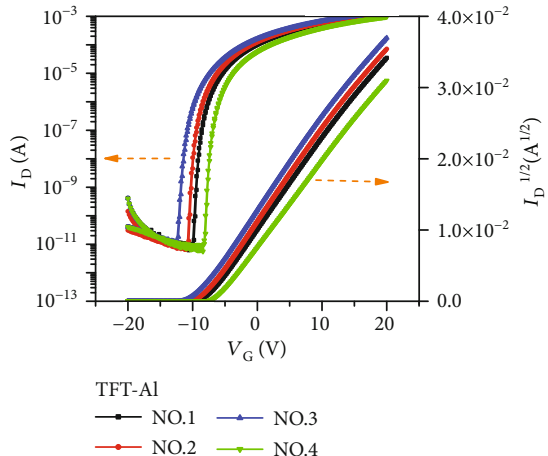

(a)

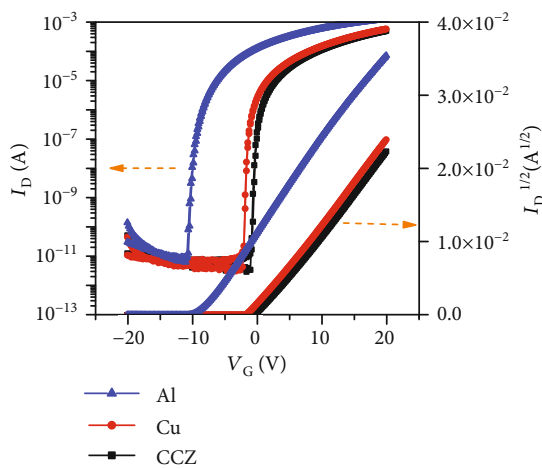

(d)

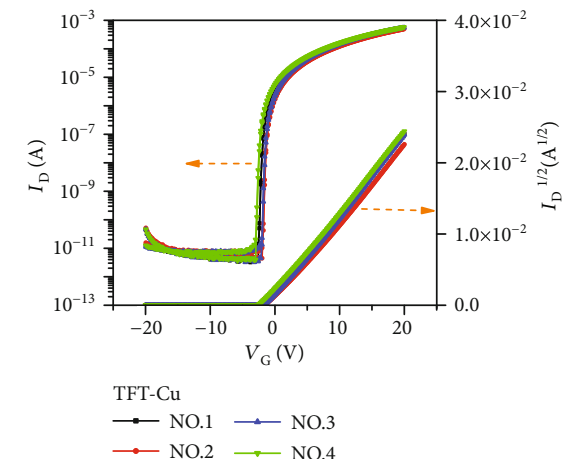

(b)

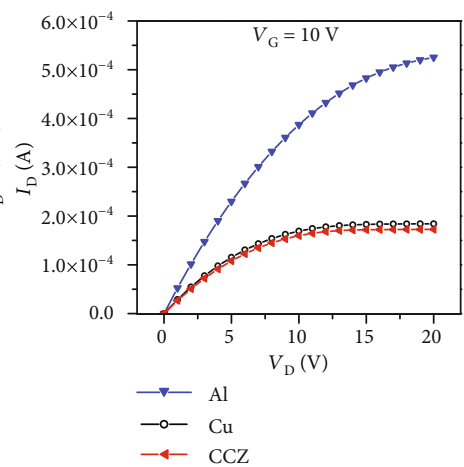

(e)

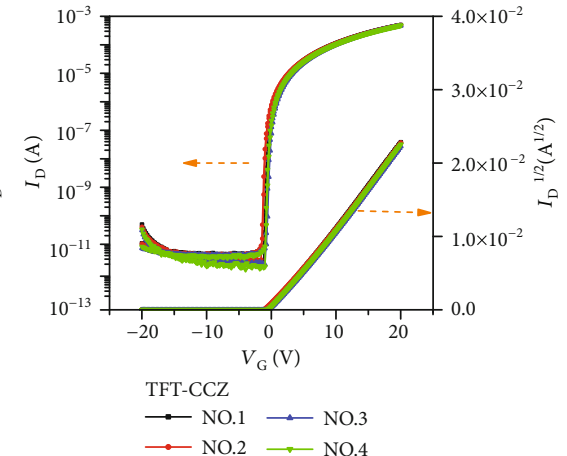

(c)

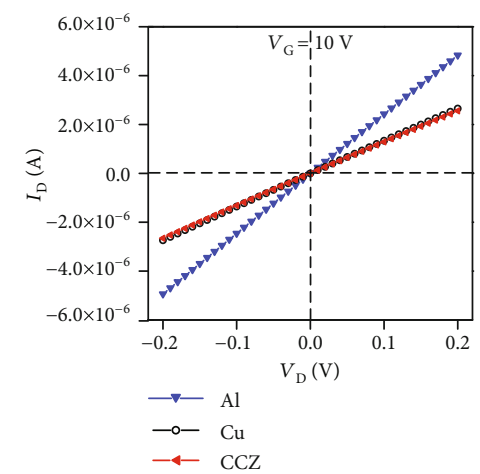

(f)

FIgure 2: Electrical characteristics of NdIZO-TFTs: transfer curves with (a) Al S/D electrode, (b) Cu S/D electrode, and (c) CCZ S/D electrode. (d) Comparison of the three kinds of S/D electrodes; output curves of (e) the whole region with $V_{\mathrm{D}}$ from $0 \mathrm{~V}$ to $20 \mathrm{~V}$ and (f) the linear region with $V_{\mathrm{D}}$ from $-0.2 \mathrm{~V}$ to $0.2 \mathrm{~V}$.

Al. And thanks to the high stability and adhesion strength of the CCZ electrode, the flexible device is so robust that it can withstand 120,000 (120k) times bending without obvious deterioration in transfer characteristics (Figure S1). Considering that the preparation conditions are completely identical, the difference of the transfer characteristics in Figure 2(d) can be ascribed to different S/D electrodes. It is interesting that TFT-CCZ shows positive threshold voltage $\left(V_{\text {th }}\right)$, which indicates enhancement working mode, while the NdIZO-TFTs with the pure $\mathrm{Cu}$ (TFT-Cu) or pure $\mathrm{Al}$ (TFT-Al) S/D electrode show negative threshold voltage $\left(V_{\mathrm{th}}\right)$, which indicates depletion working mode. Both the TFT-Al and TFT-Cu exhibit normally on switching characteristic with a negative $V_{\text {th }}$, which means residual free electron carrier transport from the source to drain electrode when without gate modulation voltage. The representative electrical parameters extracted from Figure 2(d) and parameters of NdIZO-TFT from other literatures are shown in Table $1[38,39]$. On the whole, our work shows better performance due to reasonable process parameters than other literatures. It is found that the transfer characteristics of TFT-Cu and TFT-CCZ in Figure 1(d) exhibit parallel shift, resulting in the almost identical electrical parameters. While the mobility of TFT$\mathrm{Al}$ is much higher than that of TFT-Cu and TFT-CCZ, the latter two exhibit improved SS and $V_{\text {th }}$ values; moreover, the TFT-CCZ reveals further improvement of the threshold delay to an enhancement type. Since the only difference of the TFT devices is the S/D electrode material, the contact between the $S / D$ electrode and the active layer plays a critical role in carrier injection.

The output characteristics of NdIZO-TFT with different electrodes are shown in Figure 2(e). The output current of TFT-Al is much higher than that of TFT-Cu and TFT-CCZ under the same gate voltage $\left(V_{\mathrm{G}}\right)$ modulation, which reveals that the S/D electron injection for TFT-Al is much easier than that for the other two devices. There are two possible mechanisms to suppress the injection of electron: (i) potential barrier formed by the Schottky contact and (ii) electron block layer to reduce the conductivity of the contact region. Figure 2(f) shows the linear region $I-V$ characteristics in the low-drain voltage regime under $V_{\mathrm{G}}=10 \mathrm{~V}$. The $I-V$ linear relation without current crowding phenomenon indicates a totally ohmic contact between any kind of above electrodes and the NdIZO active layer.

\subsection{The Interface Contact Characteristics between NdIZO} and Different $S / D$ Electrodes. The work functions of the electrodes were extracted from UPS spectra in Figure 3(a). As shown in the schematic diagram of the energy levels in Figure 3(b), the work functions of these three electrodes are all higher than the Fermi level of the active layer, corresponding to the ohmic contact demonstrated in Figure 3(b). In particular, the work function of CCZ is closest to the Fermi level 
TABLE 1: Electrical parameters of above devices annealed at $360^{\circ} \mathrm{C}$ and parameters of NdIZO-TFT from other literatures.

\begin{tabular}{lcccrr}
\hline S/D electrode & $I_{\mathrm{ON}} / I_{\mathrm{OFF}}$ & $\mu_{\text {sat }}\left(\mathrm{cm}^{2} \cdot \mathrm{V}^{-1} \cdot \mathrm{s}^{-1}\right)$ & $\mathrm{SS}\left(\mathrm{V} \cdot \mathrm{dec}^{-1}\right)$ & $V_{\text {th }}(\mathrm{V})$ & Notes \\
\hline Al & $1.93 \times 10^{8}$ & 52.6 & 0.23 & -8.5 & -1.1 \\
$\mathrm{Cu}$ & $1.79 \times 10^{8}$ & 40.8 & 0.10 & 0.83 & This work \\
$\mathrm{CCZ}$ & $1.24 \times 10^{8}$ & 40.3 & 0.12 & -4.74 & This work \\
ITO & $\sim 10^{7}$ & 30.4 & 0.26 & -0.97 & Ref. [38] \\
ITO & $\sim 10^{6}$ & 4.25 & 0.34 & Ref. [39] \\
\hline
\end{tabular}

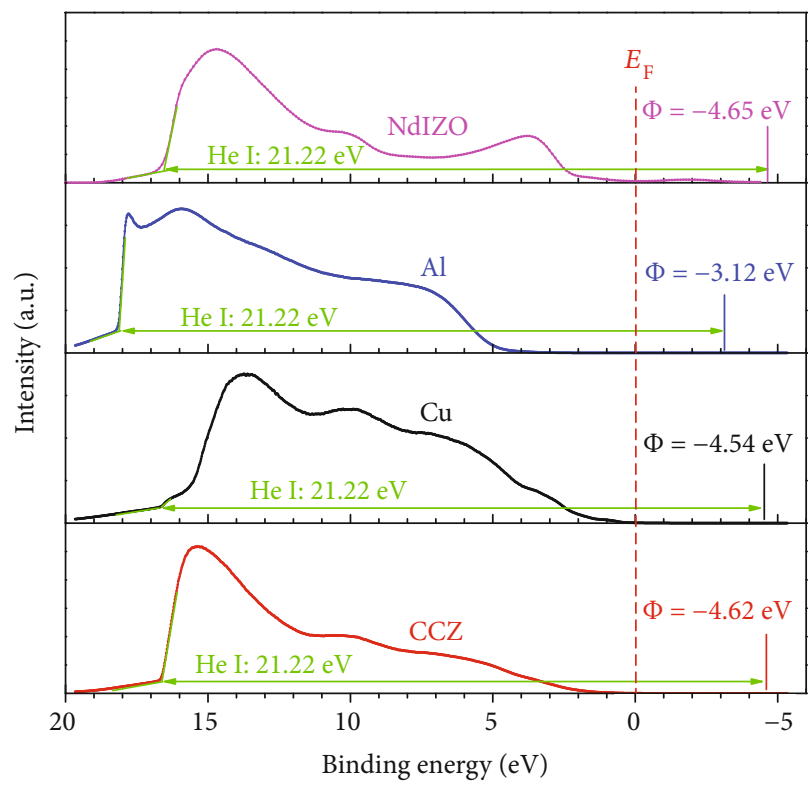

(a)

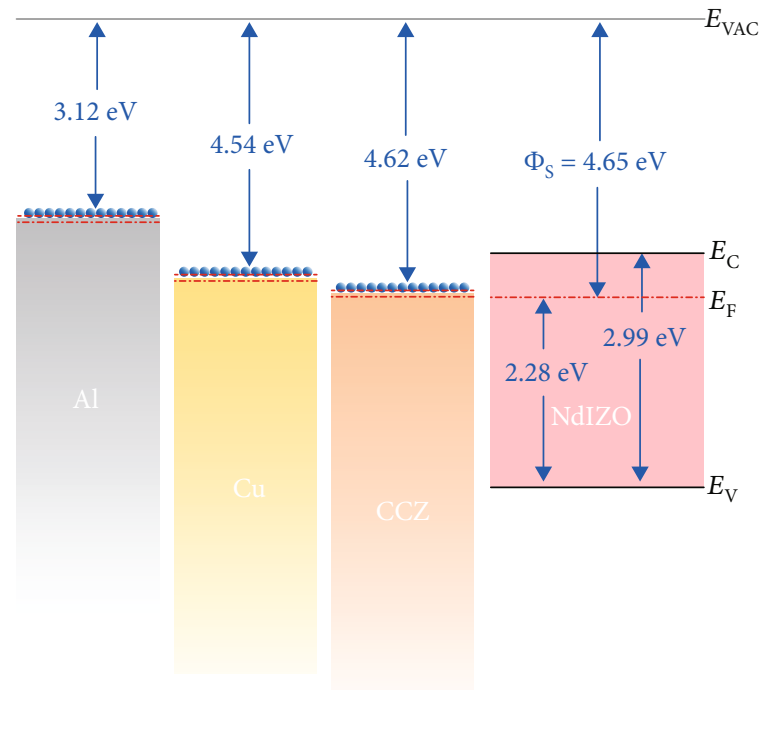

(b)

FIgURE 3: The work functions of the electrodes and active layer: (a) the UPS spectra of NdIZO, Al, Cu, and CCZ; (b) the schematic diagram of the energy level of the electrodes and active layer before contact.

of NdIZO, indicating a potential of good contact match with that of the active layer. Hence, the possibility of the Schottky contact rectification for TFT-Cu and TFT-CCZ is excluded.

Although the ohmic contact of above electrodes is substantiated, there is no powerful evidence to prove that the electrical changes of the oxide semiconductor-based TFTs with different S/D materials are determined directly by work function of the S/D electrode, because of the high carrier concentration of the AOS [40]. Hence, the contact resistance $\left(R_{\mathrm{C}}\right)$ with different $\mathrm{S} / \mathrm{D}$ electrode materials was analyzed by using the transmission line model (TLM) [41], as shown in Figures $4(\mathrm{a})-4(\mathrm{c})$. The total resistance $\left(R_{\mathrm{T}}\right)$ of the device is equal to the sum of the channel resistance $\left(r_{\mathrm{ch}} L\right)$ and $R_{\mathrm{C}}$, where $R_{\mathrm{C}}$ can be expressed through $L_{\mathrm{T}}$ (the current transfer length), as shown in

$$
R_{\mathrm{T}}=\frac{V_{\mathrm{D}}}{I_{\mathrm{D}}}=r_{\mathrm{ch}} L+R_{\mathrm{C}}=r_{\mathrm{ch}} L+r_{\mathrm{ch}} \cdot 2 L_{T},
$$

where $r_{\mathrm{ch}}$ is the channel resistance per unit channel length $(L)$. In the TLM plot, the horizontal coordinate of the cross point is equal to $2 L_{\mathrm{T}}$ and the vertical coordinate is equal to $R_{\mathrm{C}}$. In Figures 4(a) and 4(b), the fitted lines do not intersect at one point, and each intercept of the ordinate represents the $R_{\mathrm{C}}$ under a specific $V_{\mathrm{G}}$. In Figure $4(\mathrm{c})$, the fitted lines intersect at one point, resulting in constant $R_{\mathrm{C}}$, which is similar to the contact situation of Si-based TFTs with a highly doped ohmic $\left(n^{+}\right)$region below the S/D electrodes. This similarity can be ascribed to the very low work function of $\mathrm{Al}$. The comparison of $R_{\mathrm{C}}$ for different $\mathrm{S} / \mathrm{D}$ electrodes is shown in Figure $4(\mathrm{~d})$. The results show that both the $R_{\mathrm{C}}$ of TFTCCZ and TFT-Cu will be modulated with the variation of $V_{\mathrm{G}}$ : low $V_{\mathrm{G}}$ corresponding to high $R_{\mathrm{C}}$ and high $V_{\mathrm{G}}$ corresponding to low $R_{\mathrm{C}}$. Therefore, it can be speculated that the relatively high $R_{\mathrm{C}}$ of TFT-CCZ under low $V_{\mathrm{G}}$ is conducive to suppress the injection of electron in the subthreshold regime. When the $V_{\mathrm{G}}$ operates at high level, the $R_{\mathrm{C}}$ decreases rapidly, even smaller than the constant $R_{\mathrm{C}}$ of TFT-Al, thus insuring efficient injection of electron. It is believed that the modulation of $R_{\mathrm{C}}$ by $V_{\mathrm{G}}$ is the key to the realization of high-quality switching characteristic.

It is worth noting that the $R_{\mathrm{C}}$ of TFT-CCZ is always higher than that of TFT-Cu, which may be attributed to the interface reaction caused by the alloying of copper metal. To verify this conjecture, a double-layer sample of $\mathrm{CCZ} / \mathrm{NdIZO}$ was prepared, and the thicknesses of the CCZ 


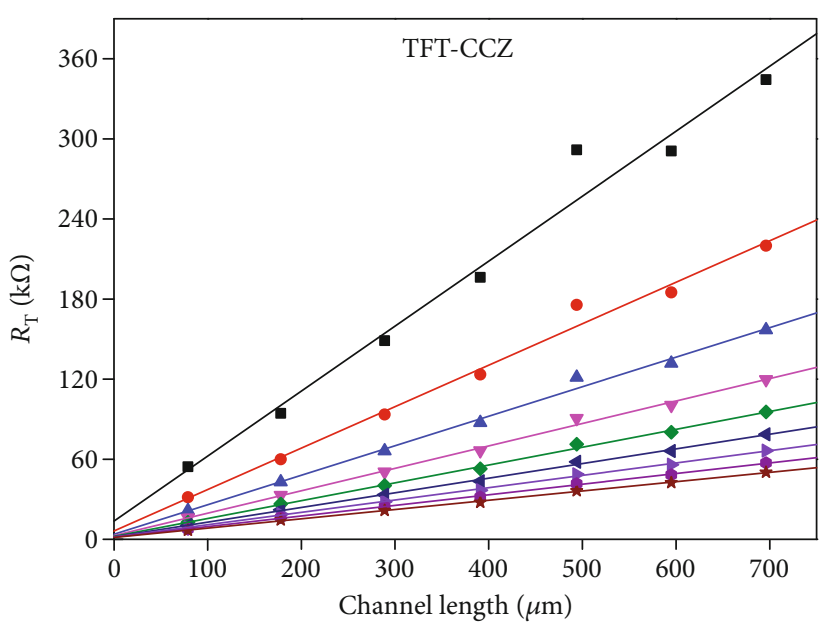

(a)

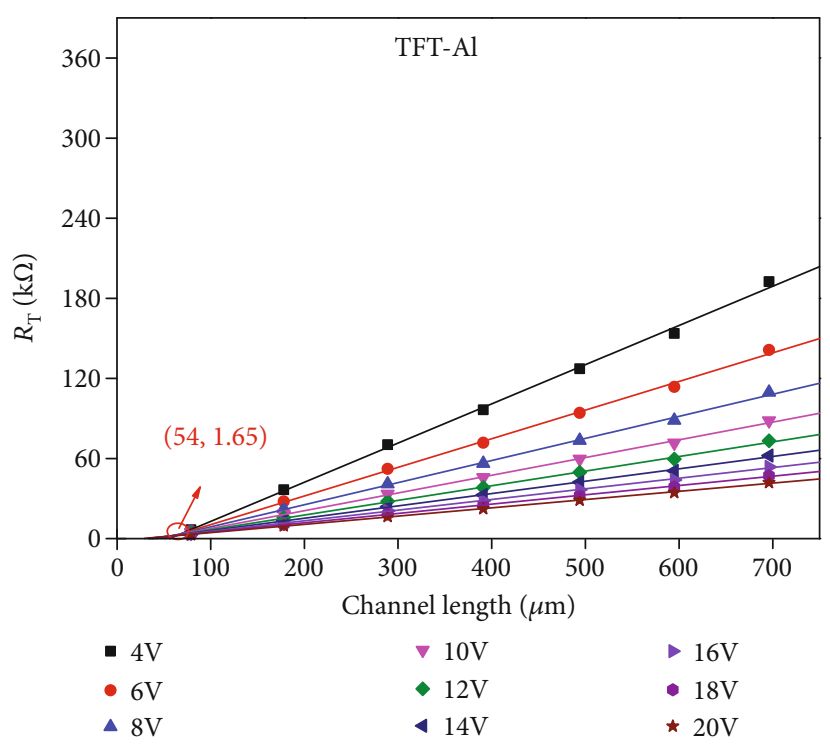

(c)

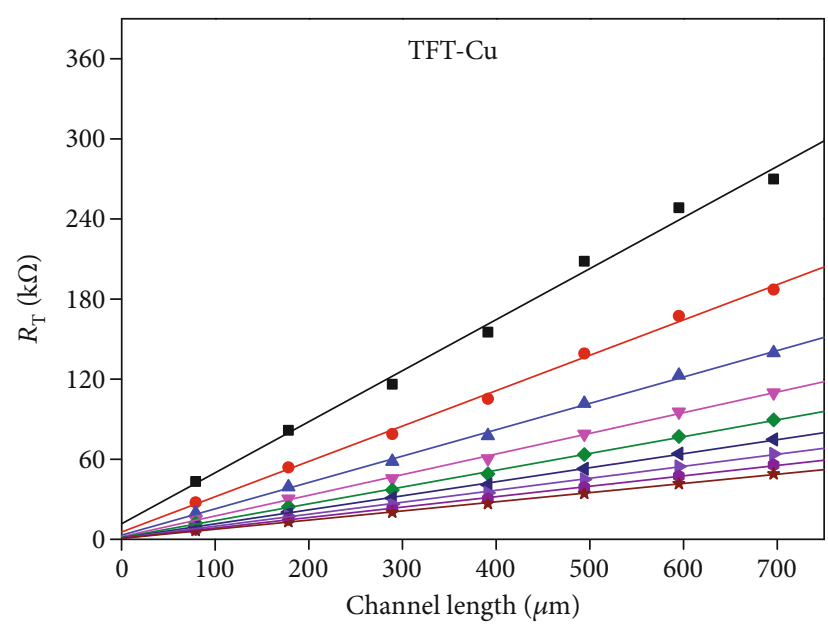

(b)

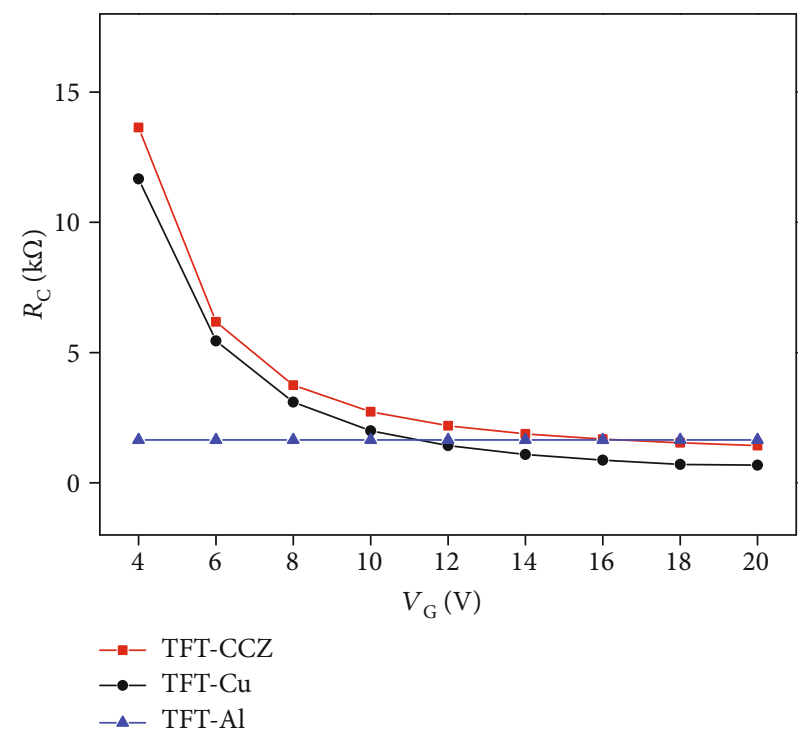

(d)

Figure 4: $R_{\mathrm{T}}$ as a function of channel length with different $V_{\mathrm{G}}$ for NdIZO-TFTs with (a) CCZ, (b) pure Cu, and (c) pure Al S/D electrodes. (d) Comparison of $R_{\mathrm{C}}$ for different $\mathrm{S} / \mathrm{D}$ electrodes.

and NdIZO were $100 \mathrm{~nm}$ and $200 \mathrm{~nm}$, respectively. The deposition and annealing parameters were identical with the TFT-CCZ device. XPS depth profile analysis of the sample was performed. Figure 5 shows the element distribution by XPS depth profile analysis, and the inset shows the interface between the CCZ S/D electrode and NdIZO active layer. It can be seen that $\mathrm{Zr}$ and $\mathrm{Cr}$, as refractory metals, do not form intermetallic compounds with $\mathrm{Cu}$ and segregate on the surface and interface, which is consistent with our previous work [42]. Moreover, this morphology acting as a self-aligned barrier buffer layer can effectively block the diffusion of $\mathrm{Cu}$ atoms into the NdIZO active layer, thereby preventing the activity of the NdIZO active layer from being affected by the $\mathrm{Cu}$ deep-level acceptor trap. In addition, the emergence of the In component before the $\mathrm{Zn}$ component indicates an In-rich region at the interface, which can be ascribed to the bombard- ment effect during CCZ deposition that breaks the weak In bond [43]. Besides, the steep profile of $\mathrm{Cu}$ in the NdIZO layer reveals an effective suppression of $\mathrm{Cu}$ atom diffusion by the interface segregation of $\mathrm{Zr}$ and $\mathrm{Cr}$.

To further investigate the chemical states of the $\mathrm{Cr}$ and $\mathrm{Zr}$ elements segregated at the contact interface, $\mathrm{Cr} 2 \mathrm{p}$ and $\mathrm{Zr} 3 \mathrm{~d}$ spectra from the CCZ/NdIZO interface were analyzed. As shown in Figure 6(a), the Cr 2p peak can be fitted by three nearly Gaussian distributions, which are centered at $573.6 \mathrm{eV}, 575.8 \mathrm{eV}$, and $577.3 \mathrm{eV}$. The three peaks are generally attributed to metal and suboxide. Figure 6(b) shows the $\mathrm{Zr} 3 \mathrm{~d}$ peak fitted by two nearly Gaussian distributions, which are centered at $182 \mathrm{eV}\left(\mathrm{Zr} \mathrm{3d}_{5 / 2}\right)$ and $184.4 \mathrm{eV}\left(\mathrm{Zr} \mathrm{3d_{3/2 }}\right)$. The $\mathrm{Zr} 3 \mathrm{~d}$ peaks determine that $\mathrm{Zr}$ exists in the form of oxide $\left(\mathrm{ZrO}_{2}\right)$ at the interface. As a result, an oxide layer from $\mathrm{Cr}$ and $\mathrm{Zr}$ is confirmed at the contact interface, which accounts for higher $R_{\mathrm{C}}$ of TFT-CCZ. 


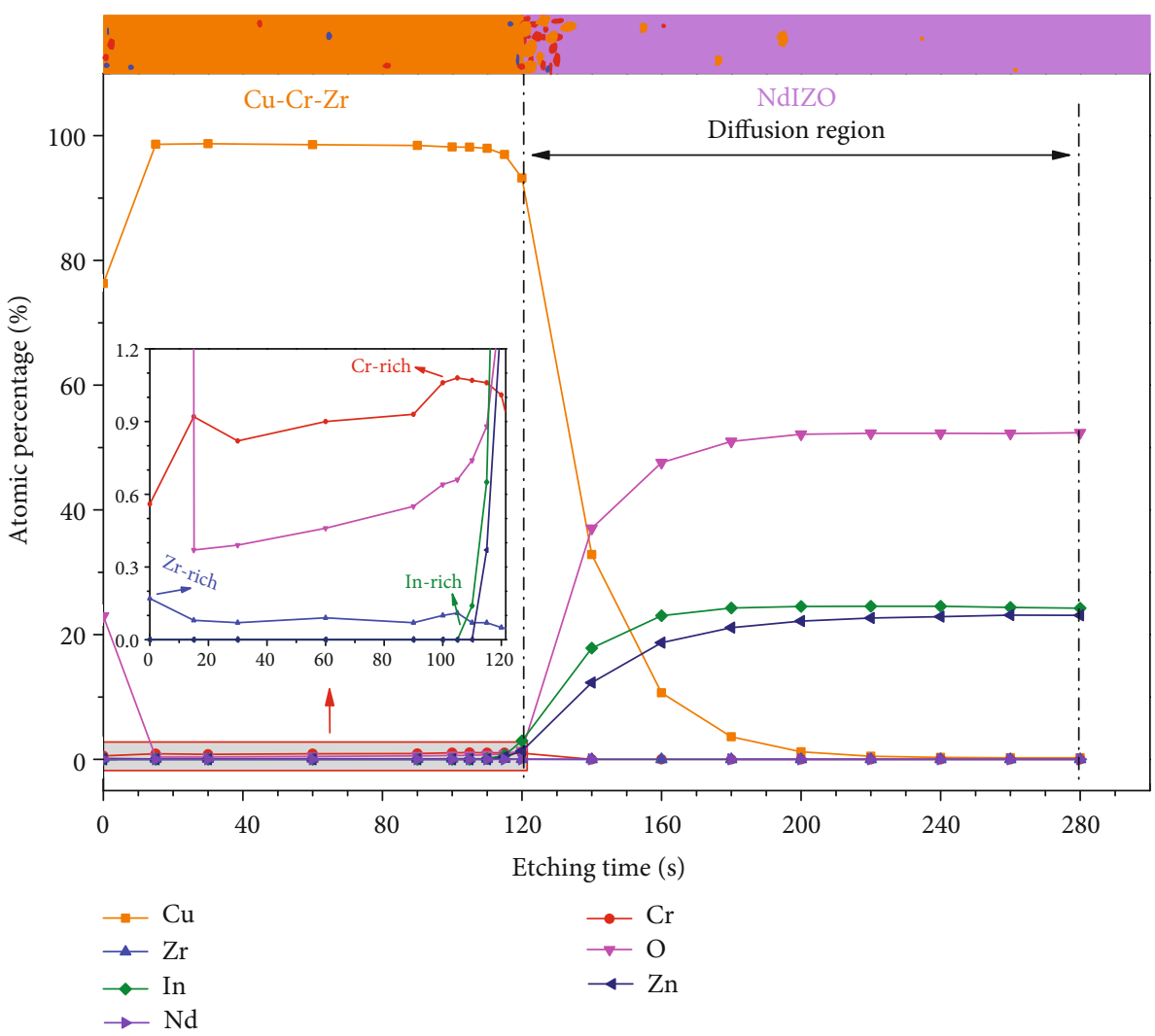

FIGURE 5: The element distribution of CCZ/NdIZO structure.

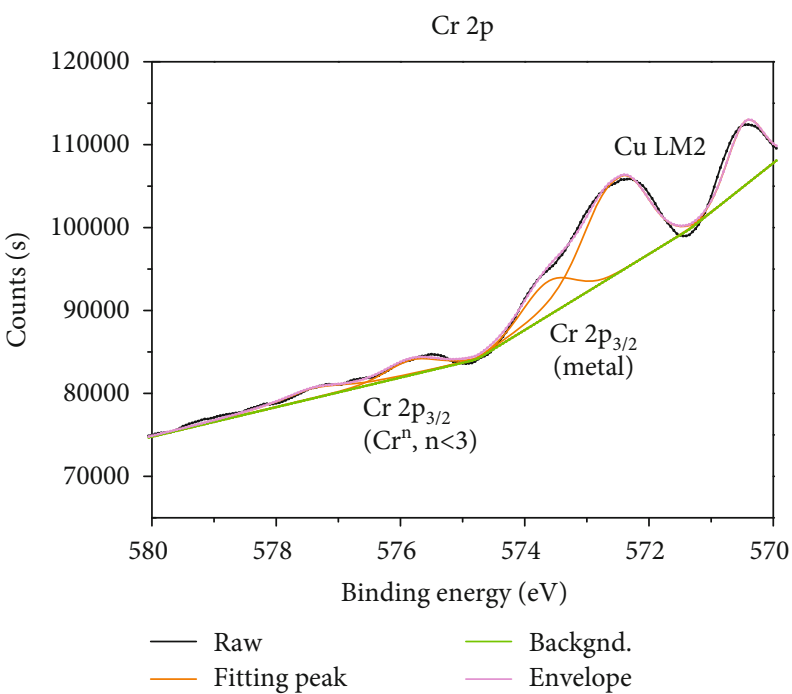

(a)

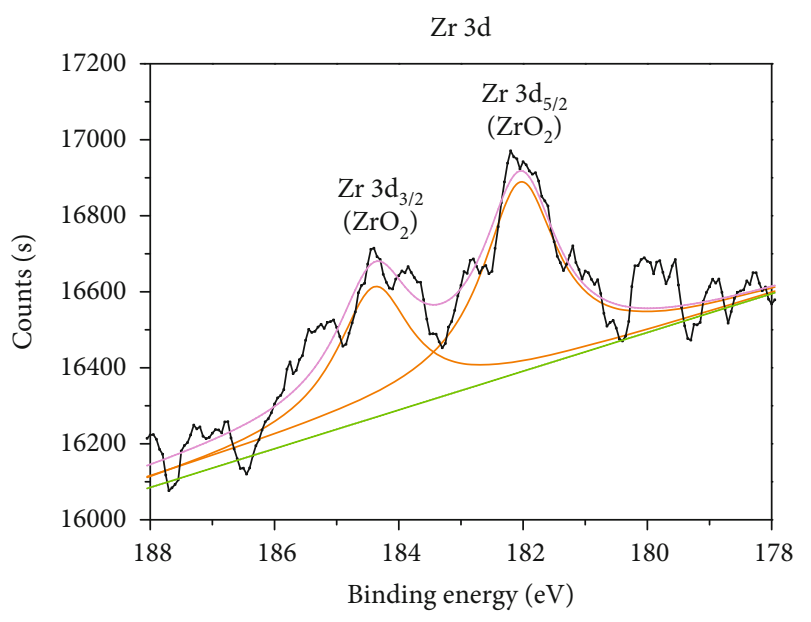

(b)

Figure 6: The XPS spectra for (a) Cr 2p and (b) Zr 3d at the interface of the CCZ/NdIZO sample.

On the basis of the above analyses and results, a hypothetic energy band diagram of carrier transport between the source and drain is proposed to explain why different S/D electrodes will affect carrier injection dramatically. As shown in Figure 7(a), due to the very low work function of the $\mathrm{Al}$ electrode, the end of the energy band will bend significantly and form potential well through the metal-semiconductor contact effect. Thus, a very negative gate voltage is required to deplete the residual free electrons, which is equivalent to increasing the potential barrier of the conduction band. A $\mathrm{Cu}$ electrode with a higher work function will improve this situation, as shown in Figure 7(b). However, the channel still cannot be pinched off without a negative gate voltage due to the high conductivity of NdIZO that ensures high mobility. As a consequence, a self-assembled oxide block layer is introduced on the contact region with the assistance of the CCZ 


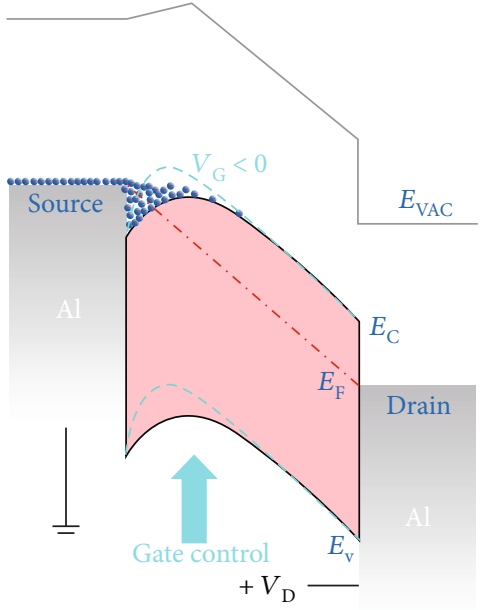

(a)

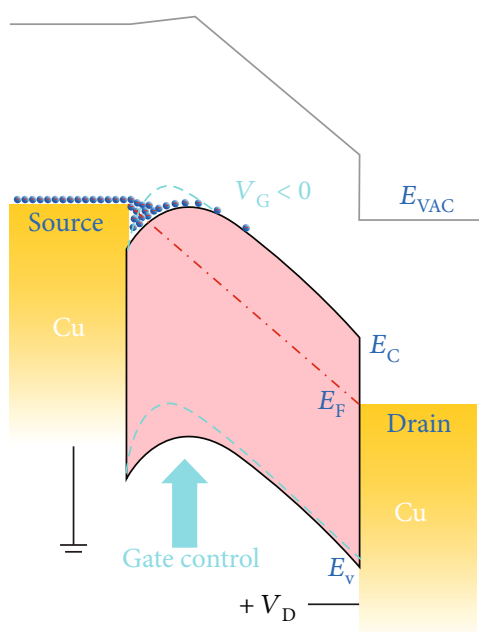

(b)

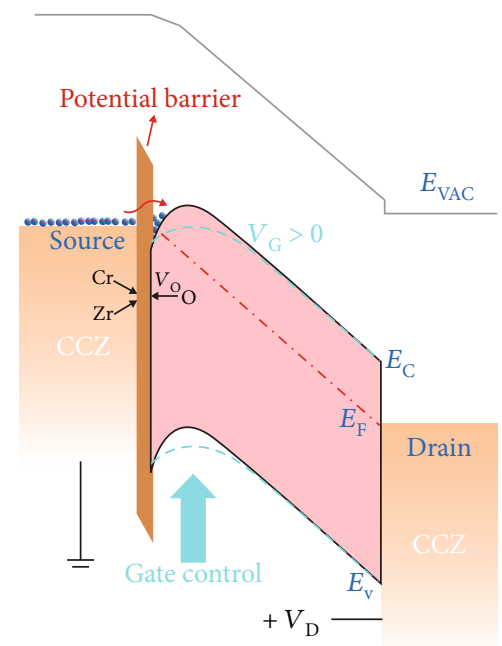

(c)

FIGURE 7: Schematic diagram of the energy band for carrier transportation from source to drain: (a) TFT-Al, (b) TFT-Cu, and (c) TFT-CCZ.

alloy electrode, as shown in Figure 7(c). The carriers need to tunnel through the additional block layer, which is equivalent to increasing the potential barrier of the conduction band. Therefore, an enhancement mode with a normally off channel is realized since a positive gate voltage is needed to turn on the channel.

\section{Conclusion}

High-performance enhancement-type flexible NdIZO-TFTs were realized by using the CCZ alloy electrode. The influence of $\mathrm{S} / \mathrm{D}$ electrodes on the electrical performance of NdIZOTFT was investigated. The $R_{\mathrm{C}}$ of TFT-CCZ is modulatable by gate voltage. Compared with $\mathrm{Al}$ and $\mathrm{Cu}$ electrode materials, the CCZ electrode will increase the $R_{\mathrm{C}}$ by the selfassembled oxide layer at the contact region, which acts as a potential barrier to suppress the residual free electron injection and ensure enhancement mode without deteriorating the electrical performance significantly. The self-assembled auxiliary interface layer that blocks residual free electron makes it possible to fabricate high-performance TFTs using more conductive semiconductor which provides a novel approach to the realization of high-performance flexible NdIZO-TFTs working in enhancement mode.

\section{Experimental Details}

4.1. Materials and Methods. As delineated in Figure 1, the NdIZO-TFT was manufactured on $18 \mu \mathrm{m}$ PI film supported by ultraflat carrier glass. The gate electrode (GE)/gate insulator (GI) was prepared by using a $300 \mathrm{~nm}$ Al-Nd alloy film that partly anodized into $200 \mathrm{~nm} \mathrm{AlO}_{x}: \mathrm{Nd} \mathrm{GI}$, as described in literatures $[44,45]$. Then, a $35 \mathrm{~nm}$ active layer was deposited at room temperature through a stencil shadow mask by radiofrequency magnetron sputtering. The specific composition of the NdIZO target is $\mathrm{Nd}_{2} \mathrm{O}_{3}: \mathrm{In}_{2} \mathrm{O}_{3}: \mathrm{ZnO}=1: 62.5$ : 36.5 wt.\%. The film deposition was carried out in $\mathrm{Ar} / \mathrm{O}_{2}$ $(100: 1)$ atmosphere at a deposition pressure of $3 \mathrm{mTorr}$ and a sputtering power of $60 \mathrm{~W}$ (i.e., $3 \mathrm{~W} \cdot \mathrm{cm}^{-2}$ ). Afterwards, the device was annealed in air atmosphere at $360^{\circ} \mathrm{C}$ for $1 \mathrm{~h}$. In the end, a $150 \mathrm{~nm} \mathrm{CCZ} \mathrm{film} \mathrm{was} \mathrm{deposited} \mathrm{to} \mathrm{act} \mathrm{as} \mathrm{S/D}$ electrodes $(L / W=360 \mu \mathrm{m} / 540 \mu \mathrm{m})$. The same fabrication process was applied to compare devices except for the S/D electrode metal materials ( $\mathrm{Al}$ and $\mathrm{Cu}$ ).

4.2. Analytical Methods. The electrical characteristics of TFT devices were measured by using a semiconductor analyzer (Agilent 4155C) in dark and air environment. The chemical changes of the channel region were detected by X-ray photoelectron spectroscopy (XPS) measurements, and the work function was detected by ultraviolet photoelectron spectroscopy (UPS) measurements (ESCALAB250Xi, Thermo Fisher Scientific, Waltham, MA, USA) at a basic pressure of $7.5 \times$ $10^{-10}$ Torr. The optical band gap was detected by using an ultraviolet spectrophotometer (SHIMADZU UV2600, SHIMADZU, Tokyo, Japan). The $R_{\mathrm{T}}$ lines were fitted by means of partial least squares regression by Origin 9 software.

\section{Data Availability}

The data used to support the findings of this study are included within the article and the supplementary materials.

\section{Conflicts of Interest}

The authors declare no conflict of interest.

\section{Authors' Contributions}

K. Lu designed and conducted the experiments. K. Lu, R. Yao, W. $\mathrm{Xu}$, and $\mathrm{H}$. Ning composed the manuscript. X. Zhang, G. Zhang, Y. Li, J. Zhong, and Y. Yang organized the data and drew the figures. J. Peng checked and approved the whole work. All authors discussed the manuscript. 


\section{Acknowledgments}

This work was supported by the National Natural Science Foundation of China (Grant Nos. 51771074, 62074059, and 22090024), Guangdong Major Project of Basic and Applied Basic Research (No. 2019B030302007), Fundamental Research Funds for the Central Universities (Nos. 2020ZYGXZR060 and 2019MS012), Guangdong Natural Science Foundation (No. 2018A0303130211), South China University of Technology 100 Step Ladder Climbing Plan Research Project (Nos. j2tw202004035, j2tw202004034, and j2tw202004095), National College Students Innovation and Entrepreneurship Training Program (Nos. 202010561001, 202010561004, and 202010561009), 2021 Guangdong University Student Science and Technology Innovation Special Fund (“Climbing Plan" Special Fund) (No. pdjh2021b0036), and Ji Hua Laboratory Scientific Research Project (X190221TF191).

\section{Supplementary Materials}

Figure S1: dynamic bending test: (a) principle of the bending machine; (b) transfer characteristics of TFT-CCZ with dynamic bending up to $120 \mathrm{k}$ times. (Supplementary Materials)

\section{References}

[1] S. P. Murarka and S. W. Hymes, "Copper metallization for ULSL and beyond," Critical Reviews in Solid State and Materials Sciences, vol. 20, no. 2, pp. 87-124, 1995.

[2] L. Lu, Y. Shen, X. Chen, L. Qian, and K. Lu, "Ultrahigh strength and high electrical conductivity in copper," Science, vol. 304, no. 5669, pp. 422-426, 2004.

[3] S. W. Lee, K. S. Cho, B. K. Choo, and J. Jang, "Copper gate hydrogenated amorphous silicon TFT with thin buffer layers," IEEE Electron Device Letters, vol. 23, pp. 324-326, 2002.

[4] K. Lu, R. Yao, S. Hu et al., "High-performance and flexible neodymium-doped oxide semiconductor thin-film transistors with copper alloy bottom-gate electrode," IEEE Electron Device Letters, vol. 39, no. 6, pp. 839-842, 2018.

[5] P. T. Liu, Y. T. Chou, C. Y. Su, and H. M. Chen, "Using electroless plating $\mathrm{Cu}$ technology for TFT-LCD application," Surface and Coatings Technology, vol. 205, no. 5, pp. 1497-1501, 2010.

[6] Z. Yu, R. Ren, J. Xue et al., "The role of oxygen in the deposition of copper-calcium thin film as diffusion barrier for copper metallization," Applied Surface Science, vol. 328, pp. 374-379, 2015.

[7] M. Haneda, J. Iijima, and J. Koike, "Growth behavior of selfformed barrier at $\mathrm{Cu}-\mathrm{Mn} / \mathrm{SiO} 2$ interface at $250-450^{\circ} \mathrm{C}$," Applied Physics Letters, vol. 90, no. 25, article 252107, 2007.

[8] H. Asanuma, T. Suzuki, and T. Kusunoki, "An investigation of optimal interfacial film condition for $\mathrm{Cu}-\mathrm{Mn}$ alloy based source/drain electrodes in hydrogenated amorphous silicon thin film transistors," AIP Advances, vol. 2, no. 2, article 22147, 2012.

[9] S. Tsukimoto, T. Kabe, K. Ito, and M. Murakami, "Effect of annealing ambient on the self-formation mechanism of diffusion barrier layers used in $\mathrm{Cu}(\mathrm{Ti})$ interconnects," Journal of Electronic Materials, vol. 36, no. 3, pp. 258-265, 2007.
[10] S. Galliano, F. Bella, M. Bonomo et al., "Hydrogel electrolytes based on xanthan gum: green route towards stable dyesensitized solar cells," Nanomaterials, vol. 10, article 1585, 2020.

[11] A. Pedico, A. Lamberti, A. Gigot et al., "High-performing and stable wearable supercapacitor exploiting rGO aerogel decorated with copper and molybdenum sulfides on carbon fibers," ACS Applied Energy Materials, vol. 1, no. 9, pp. 4440-4447, 2018.

[12] G. Piana, M. Ricciardi, F. Bella, R. Cucciniello, A. Proto, and C. Gerbaldi, "Poly(glycidyl ether)s recycling from industrial waste and feasibility study of reuse as electrolytes in sodiumbased batteries," Chemical Engineering Journal, vol. 382, article 122934, 2020.

[13] K. Senthil Kumar, P.-Y. Chen, and H. Ren, "A review of printable flexible and stretchable tactile sensors," Research, vol. 2019, pp. 1-32, 2019.

[14] N. Mariotti, M. Bonomo, L. Fagiolari et al., "Recent advances in eco-friendly and cost-effective materials towards sustainable dye-sensitized solar cells," Green Chemistry, vol. 22, no. 21, pp. 7168-7218, 2020.

[15] M. Choi, Y. Kim, and C. Ha, "Polymers for flexible displays: from material selection to device applications," Progress in Polymer Science, vol. 33, no. 6, pp. 581-630, 2008.

[16] K. Lu, J. Zhang, D. Guo et al., "High-performance and flexible neodymium-doped indium-zinc-oxide thin-film transistor with all copper alloy electrodes," IEEE Electron Device Letters, vol. 41, no. 3, pp. 417-420, 2020.

[17] R. Taheri-Ledari, K. Valadi, and A. Maleki, "High-performance HTL-free perovskite solar cell: an efficient composition of ZnO NRs, RGO, and CuInS2QDs, as electron-transporting layer matrix," Progress in Photovoltaics: Research and Applications, vol. 28, no. 9, pp. 956-970, 2020.

[18] A. Maleki, Z. Varzi, and F. Hassanzadeh-Afruzi, "Preparation and characterization of an eco-friendly ZnFe2O4@alginic acid nanocomposite catalyst and its application in the synthesis of 2-amino-3-cyano-4H-pyran derivatives," Polyhedron, vol. 171, pp. 193-202, 2019.

[19] A. Maleki, "Green oxidation protocol: selective conversions of alcohols and alkenes to aldehydes, ketones and epoxides by using a new multiwall carbon nanotube-based hybrid nanocatalyst via ultrasound irradiation," Ultrasonics Sonochemistry, vol. 40, pp. 460-464, 2018.

[20] V. Soltaninejad and A. Maleki, "A green, and eco-friendly bionanocomposite film (poly(vinyl alcohol)/TiO2/chitosan/ chlorophyll) by photocatalytic ability, and antibacterial activity under visible-light irradiation," Journal of Photochemistry and Photobiology A: Chemistry, vol. 404, article 112906, 2021.

[21] R. Lu, J. Lu, X. Wei et al., “Two-step plasma treatment designed for high-performance flexible amorphous ZnAlSnO thin-film transistors replacing thermal annealing," Advanced Electronic Materials, vol. 6, no. 8, article 2000233, 2020.

[22] S. Yue, J. Lu, R. Lu et al., "Ultrathin amorphous ZnGexSnO films for high performance ultra-thin-film transistors," Applied Physics Letters, vol. 113, no. 1, article 013504, 2018.

[23] Q. Jiang, L. Feng, C. Wu et al., “Amorphous ZnAlSnO thinfilm transistors by a combustion solution process for future displays," Applied Physics Letters, vol. 106, no. 5, article 053503, 2015. 
[24] C. Wu, X. Li, J. Lu et al., "Characterization of amorphous Si$\mathrm{Zn}-\mathrm{Sn}-\mathrm{O}$ thin films and applications in thin-film transistors," Applied Physics Letters, vol. 103, article 082109, 2013.

[25] S. Jeong, Y. G. Ha, J. Moon, A. Facchetti, and T. J. Marks, "Role of gallium doping in dramatically lowering amorphous-oxide processing temperatures for solution-derived indium zinc oxide thin-film transistors," Advanced Materials, vol. 22, no. 12, pp. 1346-1350, 2010.

[26] N. Mitoma, S. Aikawa, X. Gao et al., "Stable amorphous $\mathrm{In}_{2} \mathrm{O}_{3}$ based thin-film transistors by incorporating $\mathrm{SiO}_{2}$ to suppress oxygen vacancies," Applied Physics Letters, vol. 104, no. 10, article 102103, 2014.

[27] Y. Lee, C. H. Lee, T. Nam et al., "Hydrogen barrier performance of sputtered $\mathrm{La}_{2} \mathrm{O}_{3}$ films for InGaZnO thin-film transistor," Journal of Materials Science, vol. 54, no. 16, pp. 11145-11156, 2019.

[28] S. Oh, B. Seob Yang, Y. Jang Kim et al., "Anomalous behavior of negative bias illumination stress instability in an indium zinc oxide transistor: a cation combinatorial approach," Applied Physics Letters, vol. 101, no. 9, article 092107, 2012.

[29] M. Lee, T. I. Lee, J. Cho, W. Lee, and J. Myoung, "Improved bias stress stability of In-Ga-Zn-O thin film transistors by UV-ozone treatments of channel/dielectric interfaces," Materials Science in Semiconductor Processing, vol. 30, pp. 469475, 2015.

[30] S. Aikawa, P. Darmawan, K. Yanagisawa, T. Nabatame, Y. Abe, and K. Tsukagoshi, "Thin-film transistors fabricated by lowtemperature process based on $\mathrm{Ga}$ - and $\mathrm{Zn}$-free amorphous oxide semiconductor," Applied Physics Letters, vol. 102, no. 10, article 102101, 2013.

[31] E. Chong, Y. S. Chun, and S. Y. Lee, “Amorphous siliconindium-zinc oxide semiconductor thin film transistors processed below $150^{\circ} \mathrm{C}$," Applied Physics Letters, vol. 97, no. 10, article 102102, 2010.

[32] C.-J. Kim, S. Kim, J.-H. Lee et al., "Amorphous hafniumindium-zinc oxide semiconductor thin film transistors," Applied Physics Letters, vol. 95, no. 25, article 252103, 2009.

[33] H. W. Park, B. K. Kim, J. S. Park, and K. B. Chung, "Device performance and bias instability of Ta doped InZnO thin film transistor as a function of process pressure," Applied Physics Letters, vol. 102, no. 10, article 102102, 2013.

[34] J. S. Park, K. Kim, Y. G. Park, Y. G. Mo, H. D. Kim, and J. K. Jeong, "Novel ZrInZnO thin-film transistor with excellent stability," Advanced Materials, vol. 21, no. 3, pp. 329333, 2009.

[35] S. Aikawa, T. Nabatame, and K. Tsukagoshi, "Effects of dopants in InOx-based amorphous oxide semiconductors for thin-film transistor applications," Applied Physics Letters, vol. 103, no. 17, article 172105, 2013.

[36] K. Nomura, A. Takagi, T. Kamiya, H. Ohta, M. Hirano, and H. Hosono, "Amorphous oxide semiconductors for highperformance flexible thin-film transistors," Japanese Journal of Applied Physics, vol. 45, pp. 4303-4308, 2006.

[37] M. E. Rivas-Aguilar, N. Hernandez-Como, G. GutierrezHeredia et al., "Specific contact resistance of IGZO thin film transistors with metallic and transparent conductive oxides electrodes and XPS study of the contact/semiconductor interfaces," Current Applied Physics, vol. 18, no. 7, pp. 834-842, 2018.

[38] E. Song, L. Lan, P. Xiao et al., "Thin-film transistors with neodymium-incorporated indium-zinc-oxide semiconduc- tors," IEEE Transactions on Electron Devices, vol. 63, no. 5, pp. 1916-1920, 2016.

[39] J. Chen, X. Huang, Q. Li et al., "All-sputtering, high-transparency, good-stability coplanar top-gate thin film transistors," Applied Sciences, vol. 9, p. 83, 2018.

[40] L. Lan, M. Xu, J. Peng et al., "Influence of source and drain contacts on the properties of the indium-zinc oxide thin-film transistors based on anodic aluminum oxide gate dielectrics," Journal of Applied Physics, vol. 110, no. 10, article 103703, 2011.

[41] G. K. Reeves and H. B. Harrison, "Obtaining the specific contact resistance from transmission line model measurements," IEEE Electron Device Letters, vol. 3, no. 5, pp. 111-113, 1982.

[42] J. Peng, K. Lu, S. Hu et al., "High conductivity and adhesion of $\mathrm{Cu}-\mathrm{Cr}-\mathrm{Zr}$ alloy for TFT gate electrode," Applied Sciences, vol. 7, no. 8, p. 820, 2017.

[43] J. Park, S. Kim, C. Kim et al., "High-performance amorphous gallium indium zinc oxide thin-film transistors through $\mathrm{N}_{2} \mathrm{O}$ plasma passivation," Applied Physics Letters, vol. 93, no. 5, article 053505, 2008.

[44] Z. Lin, P. Xiao, S. Sun et al., "High-mobility thin film transistors with neodymium-substituted indium oxide active layer," Applied Physics Letters, vol. 107, no. 11, article 112108, 2015.

[45] K. Lu, R. Yao, Y. Wang et al., "Effects of praseodymium doping on the electrical properties and aging effect of InZnO thin-film transistor," Journal of Materials Science, vol. 54, no. 24, pp. 14778-14786, 2019. 